\title{
Mobile Human Network Management and Recommendation by Probabilistic Social Mining
}

\author{
Jun-Ki Min and Sung-Bae Cho
}

\begin{abstract}
Recently, inferring or sharing of mobile contexts has been actively investigated as cell phones have become more than a communication device. However, most of them focused on utilizing the contexts on social network services, while the means in mining or managing the human network itself were barely considered. In this paper, the SmartPhonebook, which mines users' social connections to manage their relationships by reasoning social and personal contexts, is presented. It works like an artificial assistant which recommends the candidate callees whom the users probably would like to contact in a certain situation. Moreover, it visualizes their social contexts like closeness and relationship with others in order to let the users know their social situations. The proposed method infers the social contexts based on the contact patterns, while it extracts the personal contexts such as the users' emotional states and behaviors from the mobile logs. Here, Bayesian networks are exploited to handle the uncertainties in the mobile environment. The proposed system has been implemented with the MS Windows Mobile 2003 SE Platform on Samsung SPH-M4650 smartphone and has been tested on real-world data. The experimental results showed that the system provides an efficient and informative way for mobile social networking.
\end{abstract}

Index Terms-Bayesian network, mobile contexts, mobile social network, SmartPhonebook.

\section{INTRODUCTION}

$\mathbf{V}$ ARIOUS social interaction services based on mobile networks are being developed as cell phones become an essential tool for communication [1]. Particularly smartphones, the high-end devices with advanced features such as Wi-Fi, Bluetooth, Global Positioning System (GPS), and gigabyte memories appear as suitable appliances in implementing the services. Users can now update their Facebook status, can send instant messages, can compose emails, and can check Twitter on their smarter mobile phones. Moreover, smartphones are becoming increasingly common. According to a recent estimate, approximately 387 million units will be shipped in 2013 , with a compound annual growth rate of $21.7 \%$ [2]. In this regard, social networking prototypes on Internet-based systems have recently focused on the mobile environments [3].

Manuscript received February 11, 2010; revised July 13, 2010 and November 4, 2010; accepted November 6, 2010. Date of publication December 17, 2010; date of current version May 18, 2011. This work was supported by the Original Technology Research Program for Brain Science (20100018948) and by the Basic Science Research Program (2009-0083838) through the National Research Foundation of Korea funded by the Ministry of Education, Science and Technology. This paper was recommended by Associate Editor H. Wang.

The authors are with the Department of Computer Science, Yonsei University, Seoul 120-749, Korea (e-mail: loomlike@sclab.yonsei.ac.kr; sbcho@cs. yonsei.ac.kr)

Color versions of one or more of the figures in this paper are available online at http://ieeexplore.ieee.org.

Digital Object Identifier 10.1109/TSMCB.2010.2092424
Although the smartphones give users many convenient services, they are, basically, communication tools that intermediate between the caller and the callee on human networks. Helping users select a contact person is an important problem because the current smartphones provide limited functions for it, which makes the user experience less pleasant and productive. When users want to contact someone via mobile phones, they can use the shortcut keys, or they can directly dial the callee's phone number, which is difficult to memorize. Otherwise, they have to search the counter party's phone number from the address book application sequentially (access for each item) or manually (type the name), which is bothersome. Managing the contact list by several groups would reduce those efforts, yet specifying a group or updating should be done. In addition, sometimes, they even forget to make contact with the invitees who have appointments with them or friends whom they have not recently seen.

Some previous studies helped the user make a phone call by providing presence cues of others', which give a chance to increase the success rate of mobile phone calls. In the beginning, they had relied on the passive annotations of the user contexts. Milewski and Smith proposed the live address book, where they augmented the traditional address book with dynamic awareness information [4]. It allows users to manually update their availability and best phone contact (e.g., office, cell phone, or home) as they move about. ConNexus presented by Tang et al. [5] employs availability information interpreted from the use or nonuse of communication channels in a mobile device and suggests the most suitable one according to a simple set of rules. Schmidt et al. introduced Context-Call that shares user's condition via a remote communication in order to make the mobile phone less disturbing [6]. As the mobile phones become smarter, some of the user contexts have been automatically retrieved, and a more broad range of services has been provided. ContextContacts [7] implemented by Oulasvirta et al. on the ContextPhone platform [8] is an augmented phonebook that shares user's contexts to others. It uses awareness cues, such as another person's current location and the device status of being in the speaker or vibrator mode, to infer the interruptibility of that other person. Here, the potential of the context-based mobile communication system is shown by performing the field test. Hwang et al. proposed UbiPhone which automatically selects the ways to contact the counter party, including voice call at home or on the cell phone and short message service [9]. It also provides the group call service that connects the caller to the most preferred member among the target group. They considered the user's call rate, the social preference defined by the user, and the counter party's location. 
Previously proposed systems described so far relied on the low-level contexts which can be directly acquired from the device's states or the user's manual inputs. It is awkward to continuously update the user's situation by hand because of the transient nature of the ubiquitous environments. Moreover, the previous systems did not support user convenience for managing his or her human network.

In this paper, the SmartPhonebook, which mines the users' social networks in order to manage their relationships by inferring social and personal contexts, is proposed. It recommends the candidate callees based on the correspondence between the user's current contexts and the past calling situations. Moreover, it visualizes the social contexts with icons and graphs so that the users can understand their social situations. It is difficult to figure out the mobile user's communication behaviors and preferences since they depend on uncertain and subjective factors. The proposed method uses Bayesian networks (BNs) to infer two kinds of contexts such as personal and social contexts in order to handle the uncertainties. The user's personal states, like emotion and behavior derived from the mobile logs, are regarded as the personal context. On the other hand, the social context such as the relationship and closeness between the user and others on the social network is extracted by mining the user's contact patterns.

Callee recommendation based on the user's situation is feasible because, in social network communities, the context of a person is strongly correlated with whom he or she is currently connecting with [10]. In other words, the user can contact different people depending on the current action or vice versa. The recommendation function of the proposed method was tested on the reality mining (RM) data set [11], while the usefulness of the method on social relationship management was verified based on the subjective test with mobile implementation.

\section{RELATED WORK}

\section{A. Mobile Context Awareness}

In the early stage of mobile context awareness, locationbased services with a simple mapping of the GPS or cell ID into semantic labels have been presented. For example, iCAMS2 [12] is a communication system where the phonebook of a handheld phone shows the information about the locations of friends, while Friendzone [13] offers the information of the friends' proximity, with the manually entered profiles relating availability to instant messaging.

Recently, various kinds of device logs and user annotated contexts have been exploited with diverse purposes. Bentley and Metcalf [14] explored the influence of context sharing in mobile environments based on the user's annotated contexts such as the location, activity, and availability. Miluzzo et al. [15] presented a personal sensing system called as CenceMe which classifies the users' physical states based on the mobile-equipped sensors such as the microphone, Bluetooth, accelerometer, and GPS. The sensed contexts can be shared through social networking applications.

Since the mobile devices are used in dynamically varying situations, many researchers have tried to discover complex contexts with advanced approaches. Hwang and Cho [16] extracted the landmarks from life logs such as the user's state, emotion, and activity by using modular BNs. There are also studies focusing on the user's physical activity such as walking, running, resting, and sleeping by using pattern recognition techniques with the mobile built-in accelerometer [17] or a set of wearable sensors connected to a mobile phone [18], [19].

\section{B. Mobile Social Network Mining}

Social network systems for mobile environments are an emerging trend both in social and ubiquitous computing. In order to support the systems, revealing of mobile social relationships and their connection structure has to be preceded. Jung [20] tried to discover the social network by using ontology reasoning. He defined the social components as tuples of the ontology and probed simple relationships like isFamilyWith and isFatherOf based on the phone subscribers' registration profiles such as the last name and age. The RM project of the MIT Media Laboratory observed interactions among 100 users by using Bluetooth-enabled smartphones over the course of nine months [21]. In addition, the users' friendship network was extracted by using the Gaussian mixture model [11], and the network and its social activities were visualized for additional analysis [22]. Miklas et al. [23] used the RM data set to investigate the effect of people's social interactions on the mobile systems, where they considered two types of relationships such as friend and stranger.

In our previous work, mobile social network mining has been conducted [24]. As the component of the social network, we have estimated the social contexts, like closeness and relationship, among users from mobile phone logs, where the mining results can be used in recommending callees or representing the user's social state.

\section{SMARTPHONEBOOK}

The proposed method helps a user maintain social relationships by visualizing the current state of the relationships and by recommending the proper contact list. In order to enable these features, the system is composed of three parts: $\log$ collection with preprocessing, context inference, and recommendation. Fig. 1 shows the overall procedure of the system.

\section{A. Mobile Context Inference}

The log collection module records a wide range of data from the call $\operatorname{logs}$ to the device information. We modified our previous log collector used in [16] for the proposed system. By mining the low-level observations, the high-level contexts, including social connections, can be inferred. However, there is much uncertainty such as the inaccuracy of the sensors and the unknown causal factors while attempting to recognize the user's context. Therefore, the context inference module adopts BNs to extract the user's personal and social states. In addition to them, the general contexts such as the current time, day of the week, and location are directly obtained from the preprocessed logs. 


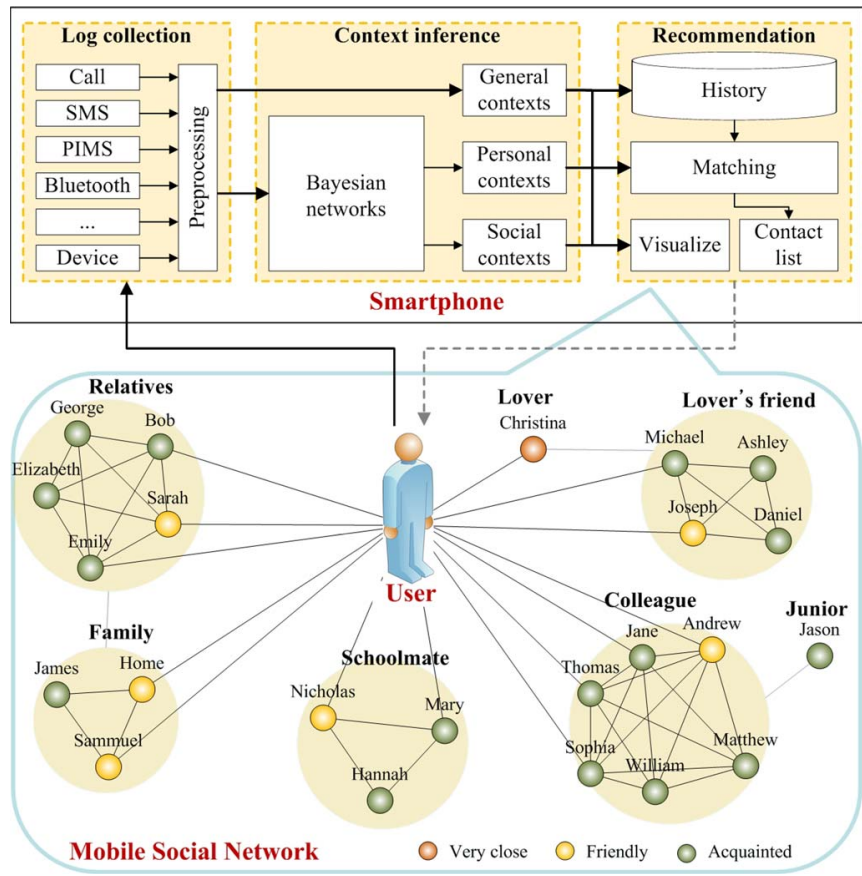

Fig. 1. Overview of the SmartPhonebook.

Each context is described more specifically in Table I. Before designing the structure and parameters of the BNs, we assumed the dependences between the high-level contexts and the logs variables as follows.

1) Emotion: Since the human mind is quite complicated, a person's emotion is difficult to know even for oneself. To make the problem simple, four types of emotional states on the valence-arousal space such as angry, sad, comfort, and joy are allowed, which is widely used for affective computing [25]. Here, we assume that the user's location, time, busyness, and current activity affect the intensity of feeling (arousal values), while the positive or negative feeling (valence values) is influenced by the activity, stress, and recent experience.

2) Busyness: A user is busy when he or she frequently contacts others having many schedules, and it would cause missed calls and unread messages.

3) Stress: Physical fatigue and psychological factors are known to cause the stress. From the information that we have collected, the discomfort index of the weather logs, the recent unfamiliar contacts, the degree of busyness, and the long periods without rest are selected as the factors.

4) Activity: In this paper, we perceive the rough states of the activity based on the user's current location, time, proximity, running application, and movement.

5) Related activity: It refers to the user's behavior during contact with the subject, where the degree of dependence with the contact is different according to the type, such as Bluetooth proximity, call, or SMS.

6) Relation type: It is connected with the public or private attributes of the contacting location and time, and the related activity, where we regard the workplace, the work hour/day, and the working activity as public; otherwise, it is private.

7) Closeness: E. Hall suggests the interpersonal distance as distinct levels of communication proximity [26]. In this paper, the degree of closeness among people is measured based on the frequency and span of contacts with different weights of the call, the SMS, and the Bluetooth proximity.

8) Relationship: In this paper, we modeled it onto the relation space that is composed of two axes, namely, the relation type and the closeness. Although there are many various situations on human relationships, their boundaries could be represented as shown in Fig. 2, in general. The overlapping regions are resolved by using the additional information on the address book, such as the last name and the address.

The Bayesian probabilistic inference is an efficient model in handling the uncertainty, and also, it easily utilizes the expert's preknowledge with its structure and parameters [27]. Let $G\left(B_{s}, \theta\right)$ be the $\mathrm{BN}$ model, where $B_{s}$ and $\theta$ denote the network structure and the set of network parameters, respectively. $\theta$ is composed of the conditional probability table $B_{\Phi}$ and the prior probability distribution $B_{p}$. For the prior probability $P(\theta)$, the knowledge discovery process is as follows:

$$
P\left(Z_{T}, Y_{T}, \Theta\right)=P\left(Y_{T} \mid Z_{T}, B_{\Phi}\right) P\left(Z_{T} \mid B_{p}\right)
$$

where $Z_{T}=\left\{z_{1}, z_{2}, \ldots, z_{T}\right\}$ represents a set of $T$ state variables and $Y_{T}$ is the corresponding observations. In this paper, we have specified $B_{s}$ and $\theta$ based on the domain knowledge, as shown in Fig. 3.

Since the BNs are based on the discrete states, variables of continuous values are needed to be quantized, which will result in some loss of information. For example, the Temperature node in Fig. 3 has to be one of the distinct states, like \{less than $0^{\circ} \mathrm{C}, 0^{\circ} \mathrm{C}-10^{\circ} \mathrm{C}, 10^{\circ} \mathrm{C}-20^{\circ} \mathrm{C}$, more than $20^{\circ} \mathrm{C}$. In order to address this problem, the proposed method dynamically changes the probability table as continuous variables based on the predefined probability function that represents the strength of confidence toward the observed event or statistical observations. For the previous example, it is possible because the node has no parent (prior conditions), as shown in Fig. 4(a).

However, if the node has parents such as the Call Stranger in Fig. 3, the evidence should be specified to a state for updating the belief to its parents. In this case, our model attaches a dummy child node [28] of binary states $\{y e s, n o\}$ having all conditional variables of the evidence node. The yes state is then chosen after setting its conditional probabilities with the observations, as shown in Fig. 4(b). Including the dummy child nodes, the personal context BN consists of 36 nodes, 40 arcs, and 83 states, while the social context $\mathrm{BN}$ has 41 nodes, 40 arcs, and 98 states, which could be easily operated on recent smartphones (for our system, the inference time took less than a second). The proposed system estimates the social contexts for each subject in the phonebook and updates after the next contact, while it periodically recalculates the personal contexts. 
TABLE I

USER'S MOBILE CONTEXTS

\begin{tabular}{|c|c|c|c|}
\hline Type & Description & Context & States \\
\hline \multirow{3}{*}{ General } & \multirow{3}{*}{$\begin{array}{l}\text { User's current state } \\
\text { directly gained from } \\
\text { the preprocessed logs }\end{array}$} & Time & $\{$ morning, afternoon, night, dawn $\}$ \\
\hline & & Day of week & $\{$ workday, weekend $\}$ \\
\hline & & Location & $\{$ home, workplace, others $\}$ \\
\hline \multirow{4}{*}{ Personal } & \multirow{4}{*}{$\begin{array}{l}\text { User's current state } \\
\text { inferred by using BN }\end{array}$} & Emotion & $\{$ angry, sad, comfort, joy $\}$ \\
\hline & & Busyness & $\{$ busy, idle $\}$ \\
\hline & & Stress & $\{$ high, low $\}$ \\
\hline & & Activity & $\{$ move, work, rest, play, others $\}$ \\
\hline \multirow{4}{*}{ Social } & \multirow{4}{*}{$\begin{array}{l}\text { Historical } \\
\text { information between } \\
\text { the user and a subject } \\
\text { inferred by using BN }\end{array}$} & $\begin{array}{l}\text { Related } \\
\text { activity }^{1}\end{array}$ & $\{$ move, work, rest, play, others $\}$ \\
\hline & & Relation type $^{2}$ & $\{$ public, private $\}$ \\
\hline & & Closeness $^{3}$ & $\{$ close, distant $\}$ \\
\hline & & Relationship & $\begin{array}{c}\text { \{family, partner, friend, colleague, } \\
\text { acquaintance, others }\}\end{array}$ \\
\hline
\end{tabular}

1. An activity that the user normally shared with the subject

2. Whether the relationship between the user and the subject is public or private

3. Whether the user has contact with the subject closely or not

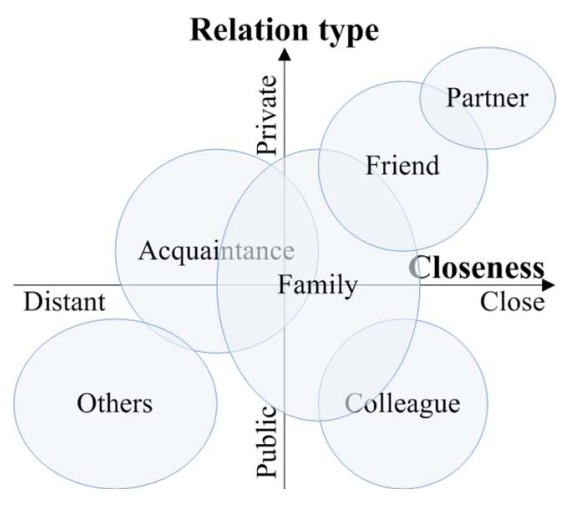

Fig. 2. Six types of relationships.

\section{B. Contact Recommendation}

Phone calls could be divided into two categories according to their purpose: calls based on individual preferences and goaldirected calls.

1) Calls based on individual preferences: The choice of the callee depends on the personal preferences and contexts of the caller without any specific reason. In this case, the calls are done for chatting or saying hello to friends or parents.

2) Goal-directed calls: It helps people act in a social behavior, such as coordinating activities or requesting help. Here, the callee is instrumental to a future goal achievement.

Generally, people tend to contact the same person whom they previously contacted in similar situations. For the goal-directed calls, the recommendation module of the proposed system statistically analyzes the user's phone call history with the past contexts and provides the candidate callee list corresponding to the user's current context. Let $p_{i}$ be the $i$ th person in the phonebook, $c_{x}$ be the $x$ th type of context, and $c_{x}(j)$ be the value of $c_{x}$ at time $j$. For the recent call list $R$, the statistical values $T$ of $p_{i}$, with the given value of the context $v\left(T\left(p_{i} \mid c_{x}()=v.\right)\right)$, are calculated as follows:

$$
T\left(p_{i} \mid c_{x}(.)=v\right)=\frac{\sum_{j=1}^{n} \operatorname{ind}\left(p_{i}, R_{j}\right) \cdot \operatorname{ind}\left(c_{x}(j), v\right)}{|R|}
$$

where $|R|$ is the number of total calls and $\operatorname{ind}(a, b)$ is an indicator function that returns one if $a$ is equal to $b$; otherwise, it returns zero. The weight $w\left(p_{i}, c_{x}\right)$, which represents the importance of $c_{x}$ for $p_{i}$, is then computed by

$$
\begin{aligned}
w\left(p_{i}, c_{x}\right) & =\sqrt{\frac{\sum_{y=1}^{\left|c_{x}\right|}\left(T\left(p_{i} \mid c_{x}(.)=v_{y}\right)-\mu\right)^{2}}{\left|c_{x}\right|}} \text { and } \\
\mu & =\frac{\sum_{y=1}^{\left|c_{x}\right|} T\left(p_{i} \mid c_{x}(.)=v_{y}\right)}{\left|c_{x}\right|} .
\end{aligned}
$$

Here, $\left|c_{x}\right|$ denotes the number of possible states of $c_{x}$, and $v_{y}$ is the $y$ th state of $c_{x}$. Finally, the score (the user's possible intention to contact $p_{i}$ on current time $t$ ) is calculated as follows:

$$
S\left(p_{i}, t\right)=\sum_{x=1}^{X} T\left(p_{i} \mid c_{x}(.)=c_{x}(t)\right) \cdot w\left(p_{i}, c_{x}\right)
$$

where $X$ refers to the number of context types. The callees of the $n$ highest scores are included in the candidate list, with a certain threshold (the number of callees to be recommended $n$ is set by the users). An example for the score calculations with seven types of contexts is shown in Table II. In order to make the problem simple, we assumed that the user made ten calls before $t$, and five times of them were to $p_{i}$. The statistical values for each state of the contexts and the weights were estimated by using (2) and (3), respectively. In this example, the score for $p_{i}$ was 0.3 . 


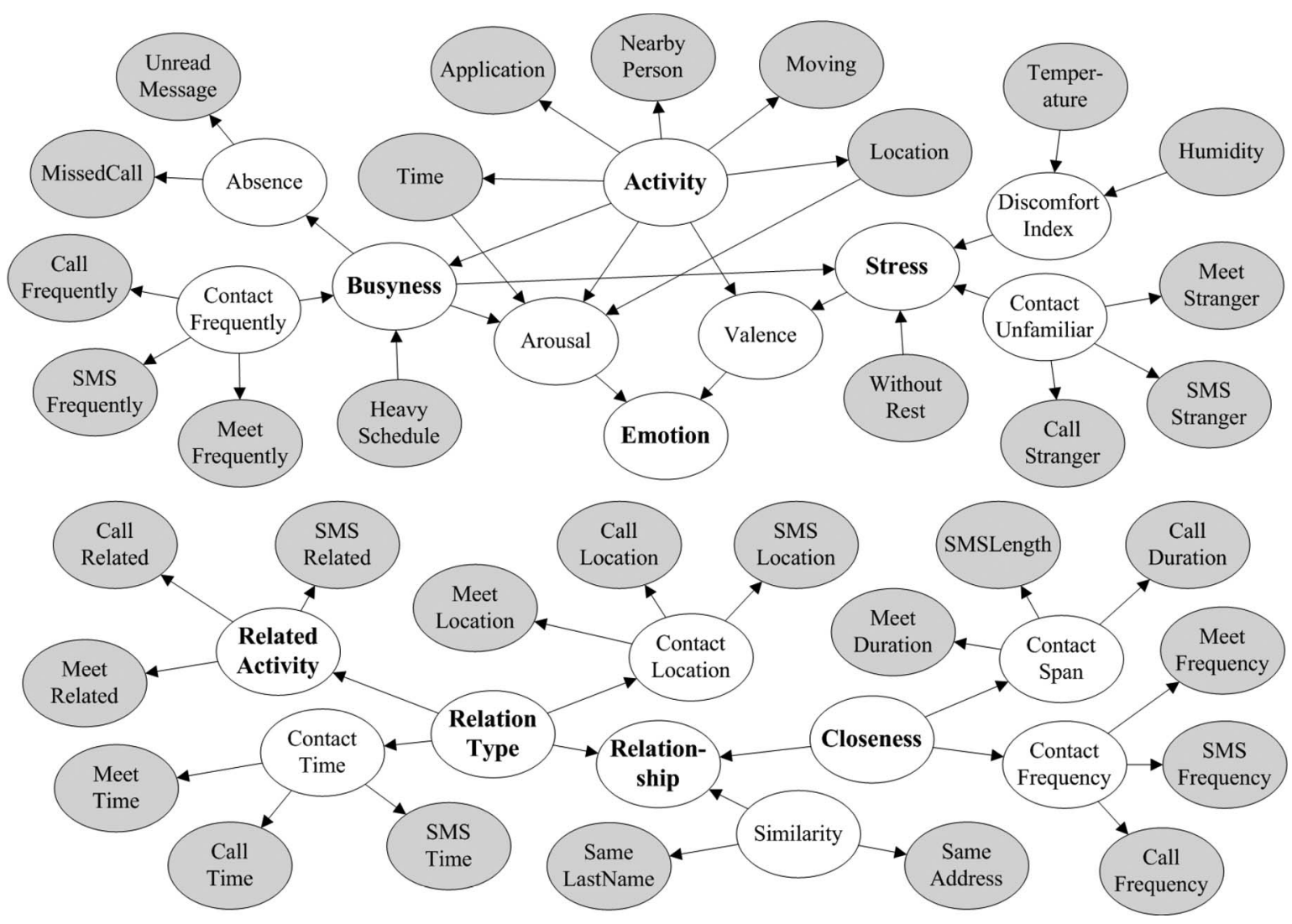

Fig. 3. BN designed for mining contexts. Gray indicates the evidence node.

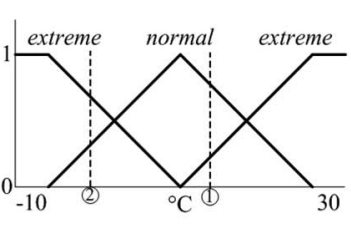

Given probability function for Temperature variables

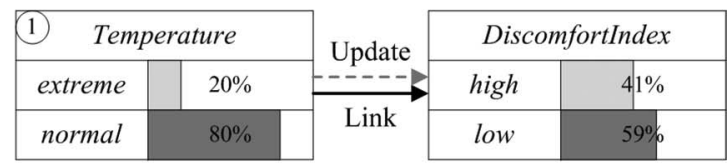

\begin{tabular}{|l|l|l|l|l|l|} 
(2) Temperature & \multicolumn{2}{|c|}{ Update } & \multicolumn{2}{c|}{ DiscomfortIndex } \\
\hline extreme & $70 \%$ & & Link & high & $63 \%$ \\
\hline normal & $30 \%$ & Link & $37 \%$ \\
\hline
\end{tabular}

(a)

Conditional probability table of Dummy_CallStranger

\begin{tabular}{|c|c|c|c|}
\hline & familiar & unfamiliar & Ensure the row sum to be \\
\hline yes & 0.7 & 0.3 & $\longrightarrow$ one, and set yes as the \\
\hline no & 0.3 & 0.7 & evidence \\
\hline
\end{tabular}

\begin{tabular}{|c|c|c|c|c|c|c|c|}
\hline \multicolumn{2}{|c|}{ Dummy_CallStranger } & \multirow{2}{*}{ Update } & \multicolumn{2}{|c|}{ CallStranger } & \multirow{2}{*}{ Update } & \multicolumn{2}{|c|}{ ContactStranger } \\
\hline yes & $100 \%$ & & familiar & $70 \%$ & & familiar & $58 \%$ \\
\hline no & $0 \%$ & & unfamiliar & $30 \%$ & & unfamiliar & $42 \%$ \\
\hline
\end{tabular}

(b)

Fig. 4. Examples of the specifying observations with continuous values where the evidence node (a) has no parents and (b) has parents.

For the preference-based calls, on the other hand, the proposed system recommends close people among whom the user has not recently contacted. Here, the degree of intimacy is the inferred social contexts, while the value of recentness has to be set by the user. This recommendation is activated whenever the user's busyness state is idle or when the number of candidates for the goal-directed calls is less than $n$.

In order to help the user understand his/her condition of social relationships, the proposed system represents the social contexts as icons, while it visualizes the social structures as a network with nodes for individuals and links for connections among people. More details are described in Section IV-A.

\section{EXPERIMENTAL RESUlts}

\section{A. Implementation}

The SmartPhonebook was implemented on the platform of the MS Windows Mobile 2003 SE Pocket PC on the Samsung 
TABLE II

EXAmple of the Score Calculation For the CALlee Recommendation

\begin{tabular}{|c|c|c|c|c|c|c|c|}
\hline & Time & Day of week & Location & Emotion & Busyness & Stress & Activity \\
\hline \multirow{5}{*}{$\begin{array}{l}\text { User's recent } \\
\text { calls to } \\
\text { person } p_{i} \\
\text { among ten } \\
\text { total calls }\end{array}$} & morning & workday & workplace & angry & busy & high & others \\
\hline & morning & workday & workplace & comfort & idle & high & work \\
\hline & night & weekend & others & comfort & busy & low & play \\
\hline & morning & workday & workplace & angry & busy & high & others \\
\hline & afternoon & workday & home & joy & idle & high & others \\
\hline $\begin{array}{c}\text { Statistical } \\
\text { values } \\
T\left(p_{i} \mid c_{x}\right)\end{array}$ & $\begin{array}{c}\text { morning }=0.3 \\
\text { afternoon }=0.1 \\
\text { night }=0.1 \\
\text { dawn }=0.0\end{array}$ & $\begin{array}{l}\text { workday }=0.4 \\
\text { weekend }=0.1\end{array}$ & $\begin{array}{c}\text { home }=0.1, \\
\text { workplace }=0.3 \\
\text { others }=0.1\end{array}$ & $\begin{array}{c}\text { angry }=0.2, \\
\text { sad }=0.0, \\
\text { comfort }=0.2, \\
\text { joy }=0.1\end{array}$ & $\begin{array}{c}\text { busy }=0.3 \\
\text { idle }=0.2\end{array}$ & $\begin{aligned} \text { high } & =0.4, \\
\text { low } & =0.1\end{aligned}$ & $\begin{array}{c}\text { move }=0.0, \\
\text { work }=0.1, \\
\text { rest }=0.0, \\
\text { play }=0.1, \\
\text { others }=0.3\end{array}$ \\
\hline $\begin{array}{l}\text { Weight } \\
w\left(p_{i}, c_{x}\right)\end{array}$ & 0.126 & 0.212 & 0.115 & 0.096 & 0.071 & 0.212 & 0.122 \\
\hline $\begin{array}{c}\text { User's } \\
\text { current } \\
\text { context } c_{x}(t)\end{array}$ & morning & workday & workplace & sad & busy & high & others \\
\hline ore $S\left(p_{i}, t\right)$ & $0.3 \times 0.126$ & $+0.4 \times 0.212$ & 0.115 & .096 & 071 & $212+$ & $122=0.3$ \\
\hline
\end{tabular}

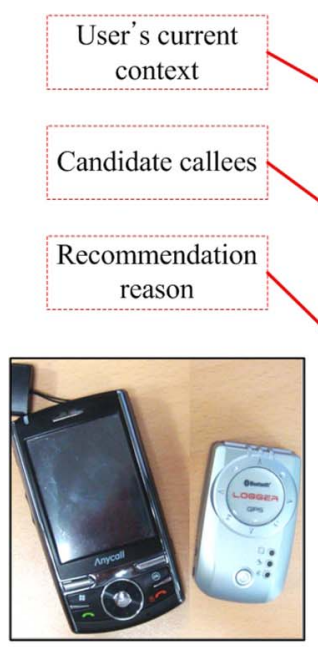

(a)

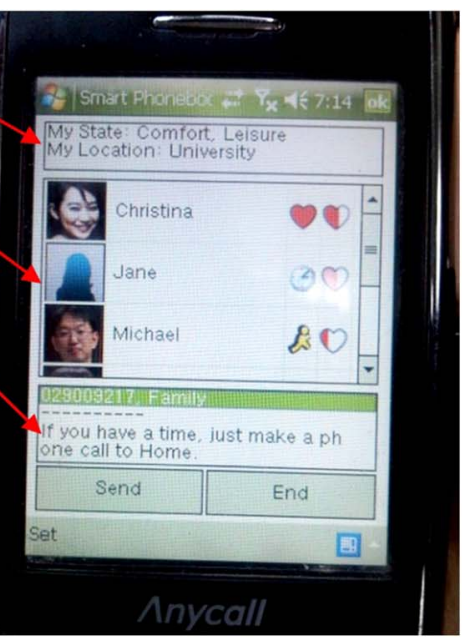

(b)

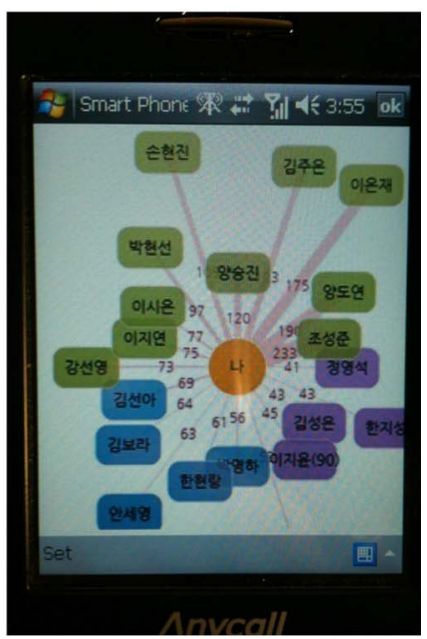

(c)

Fig. 5. (a) Mobile phone SPH-M4650 with GPS receiver used in this paper. (b) Implemented system for callee recommendation. (c) Social relationship visualization.

SPH-M4650 smartphone with a small GPS receiver that transfers data with Bluetooth to the phone [see Fig. 5(a)]. The system interface consists of three windows, as shown in Fig. 5(b): the user's current personal contexts such as emotion and the degree of busyness are displayed at the top window, the candidate callees are listed at the middle window, and the recommendation reasons for the callees are described at the bottom window.

The personal and social contexts, showed to a user and exploited for the recommendation, are automatically inferred from the mobile logs by using the proposed method. However, the user can also manually change them to more subjective states, being provided with the intended recommendation.

According to the scores explained in the previous section, persons from the user's address book are listed as the candidate callees. Here, the context type having the maximum weight [calculated by using (3)] is regarded as the major reason in recommending the corresponding person. As shown in Fig. 5(b), the icons placed at the right side of each callee depict the reason and the degree of closeness, respectively. For the given example, Jane was related to the degree of busyness or the current schedule of the user (clock icon), while Michael was involved with the current activities of the user (walking person icon). In case of Christina, she was recommended because of the user's preference (heart icon), where she and Jane were closer to the user than Michael, as illustrated by the heart gauge icons.

From the icons displayed next to the callees, a user can catch the reasons why he/she should call them at a glance. If the user did not clearly understand the meaning of the icons, he/she can confirm them using the short sentences noted at 


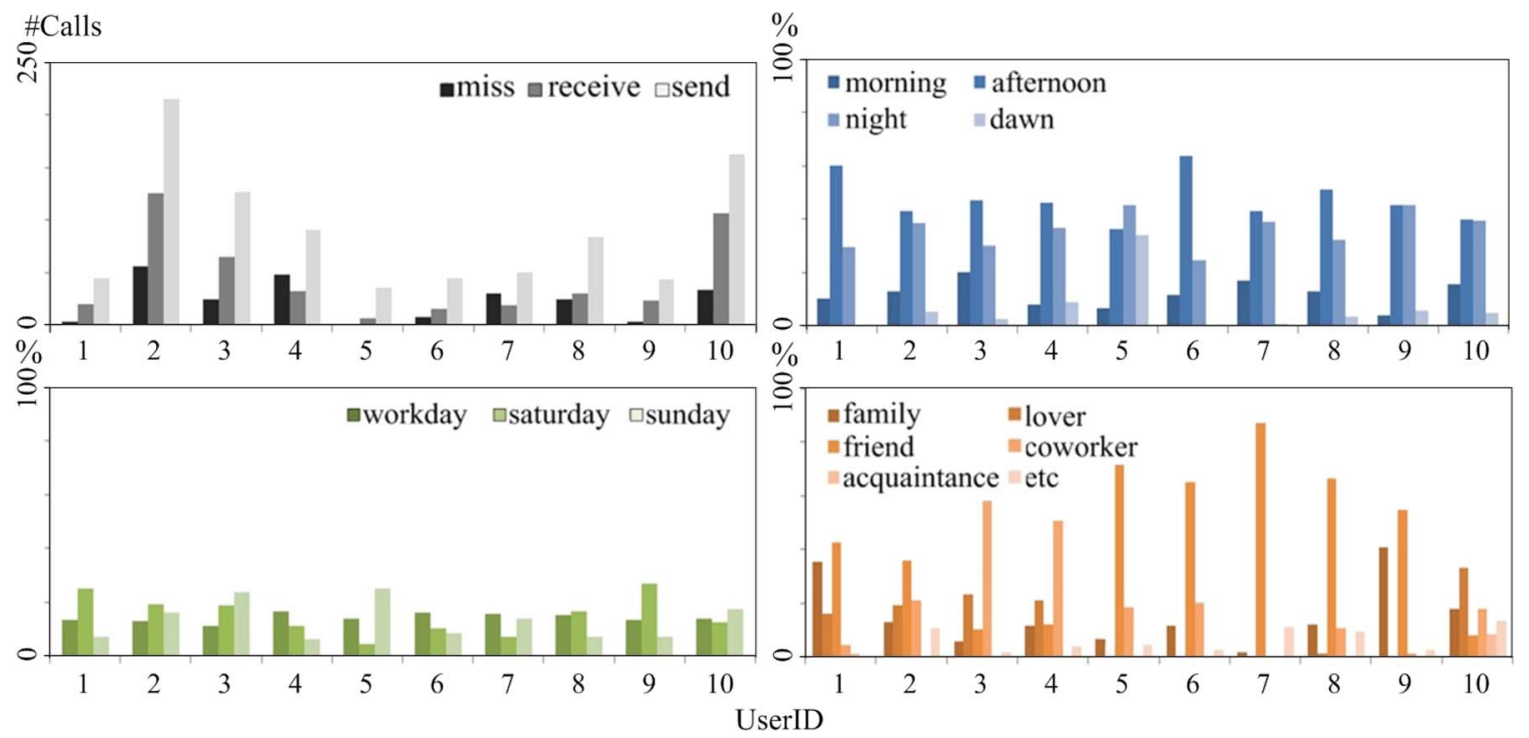

Fig. 6. Call logs collected from ten users.

the bottom window. The reason text is simply generated by replacing some keywords of the text templates with the given context states. For instance, if the inference result of the social contexts between the user and Bob is \{ Related activity = play, Closeness $=$ close, and Relationship $=$ friend $\}$, where the major reason for the recommendation is the activity context, the reason text will be generated as "You usually make a phone call with (close) (friend) (Bob) when you (play)." The reason icons and texts can help in reminding the user of the upcoming appointments (goal-directed calls) or in choosing the preferred callees.

The social contexts are also employed to visualize the state of the social network, as shown in Fig. 5(c), which is turned up on the screen when a user changes the mode in the "set" menu. The top 20 close people are shown in Fig. 5(c), where the user can change $n$, which is the number of persons to be displayed, and the criteria for displaying. The center node denotes the user, while the other nodes indicate individuals on the address book. The network represents three types of social information \{the last contact, the closeness between the user and each person, and the relationship $\}$ as the length of the link, thickness, and node color, respectively. The more time passes from the last contact with the corresponding person, the longer is the link. People who have close relations with the user are connected as thicker links, while the nodes of the same color belong to the same relationships with the user.

\section{B. Context Inference Results}

Mobile logs were collected from ten university students (User01-User10) who are 22-26 years old for over a month. Fig. 6 shows the detail of the logs, and Fig. 7 shows the correlations between the calling pattern and the inferred contexts. In this paper, attending a lecture was regarded as a work since the users were students.

Fig. 8 shows the personal contexts of User01 on a specific time and the social contexts between the user and someone else. In this case, the user was happily playing and was a bit busy (joy

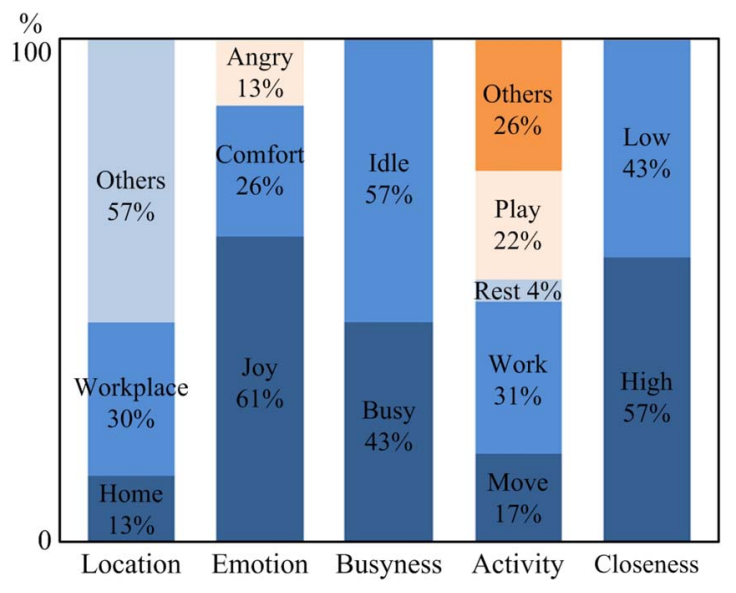

Fig. 7. Users' calling patterns on each context.

is $62 \%$, play is $87 \%$, and busy is $49 \%$ ). The user had privately contacted the subject even if they are not very closely related. Therefore, their relationship was probably a distant friend or an acquaintance, as inferred by the BN (private is $59 \%$, distant is $58 \%$, friend is $24 \%$, and acquaintance is $25 \%$ ).

We tested the accuracies of the extracted activities and relationships by comparing them with the actual labels acquired from the user reports, and we achieved about $92 \%-84.8 \%$ for the activities and $77.8 \%$ for the relationships. Here, the subjects whom the users contacted at least two times during data collection are considered for relationship verification. There were some inherent problems on the collected data in achieving higher accuracies, such as the missing personal information in the address book, which caused the confusion to the overlapping relationships. Moreover, the collection span of four weeks was insufficient in exactly modeling the human relationship. For example, if a user had contacted some friends or families only a few times for the given span, the relationship would be regarded as acquaintance or others. Fig. 9 shows an example of the changing relationships according to the accumulation of the call logs. 

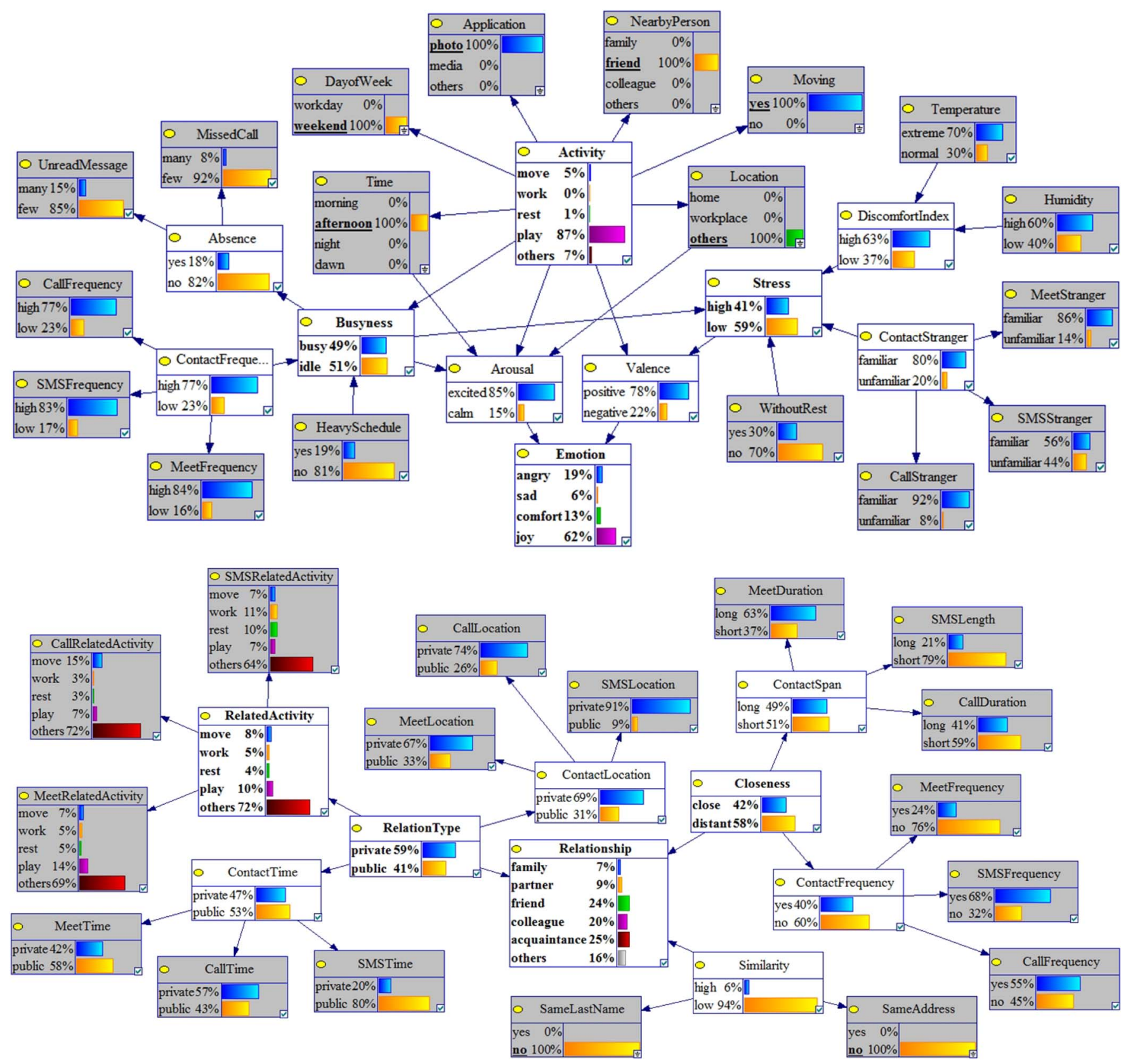

Fig. 8. Examples of the personal and social contexts inferred by the BNs.

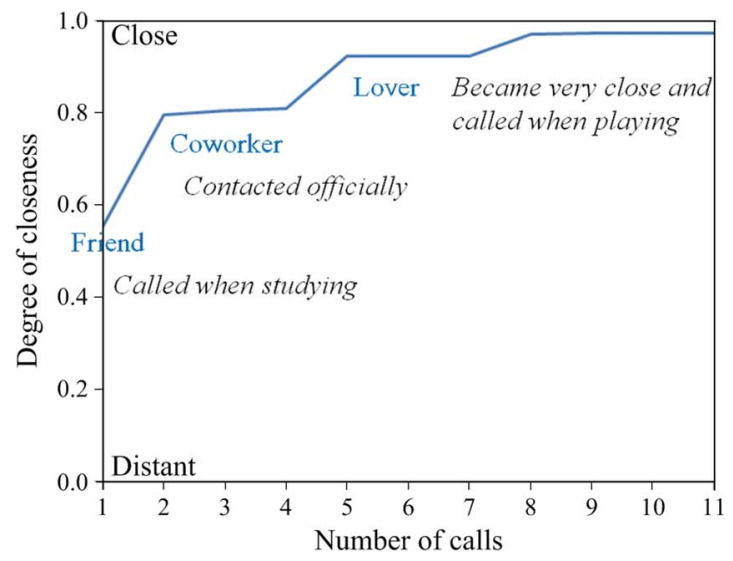

Fig. 9. Changing the user's relationships.

\section{Callee Recommendation}

The callee recommendation was validated by comparing the recommendations with the users' actual calls. The proposed system yielded about $70 \%$ accuracy, where the five callees were provided as the candidates for each recommendation. Fig. 10 shows the recommendation accuracy for each situation calculated by (\#corrects on the situation/\#calls on the situation) $\times$ $100 \%$. The preference-based calls, in case of the idle or rest state, were easy to predict since most of the targets were close people. On the other hand, knowing the users' intention for the goal-directed calls, particularly on the busy state at a workday, was difficult because of the rapidly changing dynamic factors. Moreover, a lack of logs (in our cases, at morning) can be one of the biggest obstacles of the historybased recommendation system. In this sense, the major failure 


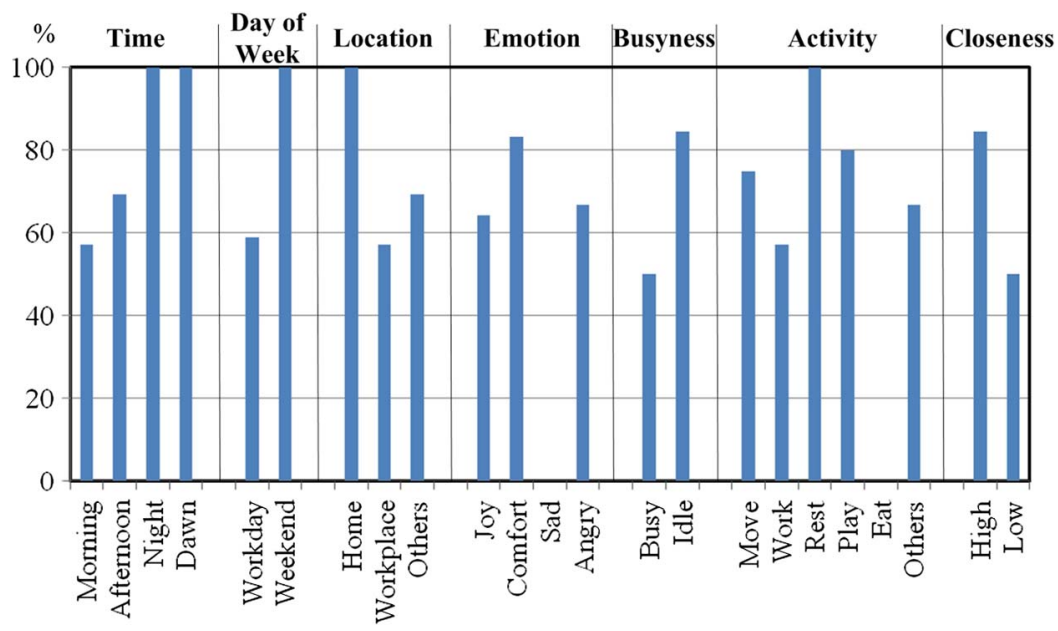

Fig. 10. Callee recommendation accuracy on each situation.
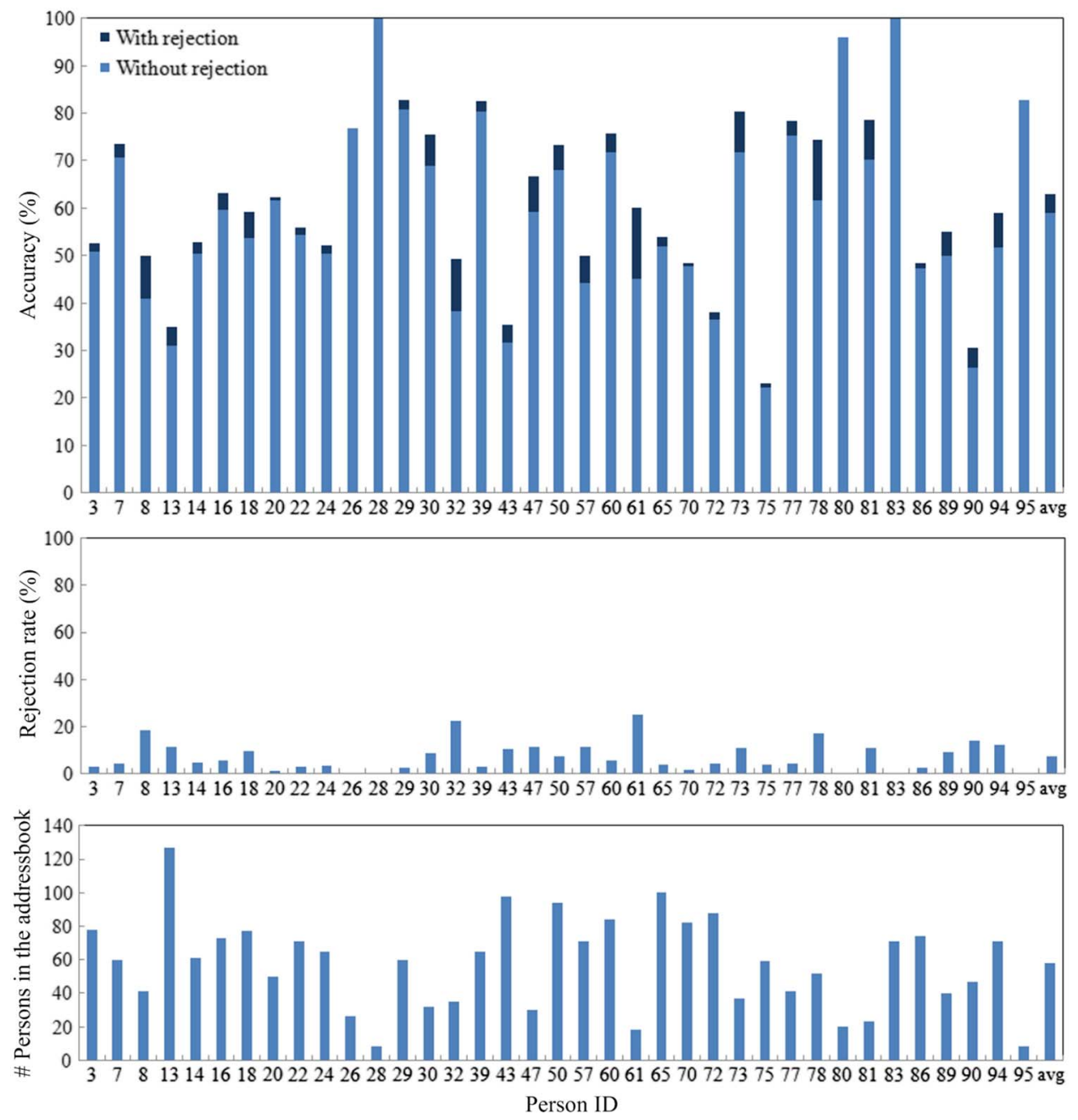

Fig. 11. Callee recommendation accuracy on the RM data set [11]. Five candidates were chosen for each suggestion from the user's address book, where the first phone call to someone is regarded as a rejection.

of the recommendation was caused by the first phone call to someone.

In order to conduct a further investigation on a larger data set, the RM data set logged from 100 subjects for nine months was employed [11], [21]. Since RM is not originally collected for callee recommendation or context inference, it does not contain sufficient types of data to apply the proposed method. Moreover, there are no user-tagged labels for social relationships or contexts so that the context inference algorithm cannot be evaluated. Therefore, we modified the probabilistic model as a simplified structure. Since the RM data set has no address book information, a pseudo address book was generated by parsing 
TABLE III

QUESTIONNAIRES OF THE SUS

\begin{tabular}{|l|c|}
\hline \multicolumn{1}{|c|}{ Questions } & Score \\
\hline 1. I think I would like to use this system frequently & $1,2,3,4,5$ \\
\hline 2. I found the system unnecessarily complex & $1,2,3,4,5$ \\
\hline 3. I thought the system was easy to use & $1,2,3,4,5$ \\
\hline $\begin{array}{l}\text { 4. I think that I would need the support of a technical person to be able to } \\
\text { use this system }\end{array}$ & $1,2,3,4,5$ \\
\hline 5. I found the various functions in this system were well integrated & $1,2,3,4,5$ \\
\hline 6. I thought there was too much inconsistency in this system & $1,2,3,4,5$ \\
\hline $\begin{array}{l}\text { 7. I would imagine that most people would learn to use this system very } \\
\text { quickly }\end{array}$ & $1,2,3,4,5$ \\
\hline 8. I found the system very cumbersome to use & $1,2,3,4,5$ \\
\hline 9. I felt very confident using the system & $1,2,3,4,5$ \\
\hline 10. I needed to learn a lot of things before I could get going with this system & $1,2,3,4,5$ \\
\hline
\end{tabular}

the phone numbers from the call logs of the data. The first year logs (2004) were adopted in estimating the probability function which represents the strength of confidence toward the observed event or statistical observations, as shown in Fig. 4. The rest of the logs (year of 2005) was used to test the algorithm, where the candidate callees were matched to the actual callee of each outgoing voice call.

As shown in Fig. 11, the proposed method achieved 63\% of recommendation accuracy for 37 subjects (there are no calls available in 2005 for the other subjects) in average, where five candidates were chosen from the user's address book. For several cases, the proposed method yielded a high accuracy of over $80 \%$, which implies the feasibility of the callee suggestion. In some cases of the users in dynamic situations, however, accuracies that are less than $40 \%$ were produced. There were two major reasons for these cases. First, it was because the user's contexts could not be precisely modeled by using the simplified BNs with the insufficient user information. Second, applying the identical model (even if it was designed for general concepts) to different persons had limitations in achieving a stable performance. In order to address the problem, personalization and adaptation should be dealt as our future work. The rejection experiment was performed for the first phone call to someone, which was the major reason for the errors in the previous experiment. Since the two problems affected much of the results, the rejection was not influential in this case.

\section{System Usability Test}

In order to validate the usefulness of the proposed method, we performed a subjective test for ten users based on the system usability scale (SUS) questionnaires. The SUS is a simple tenitem scale giving a global view of the subjective assessments of usability, where the odd-numbered items worded positively and the even-numbered items worded negatively, as shown in Table III [29]. Each item is on a five-point scale ranging from "strongly disagree" to "strongly agree," and the usability score is transformed linearly to range from 0 to 100 .

As shown in Fig. 12, the proposed system, which recommends callees and visualizes the user's social relationships, attained a higher usability score than the mobile phone's orig-

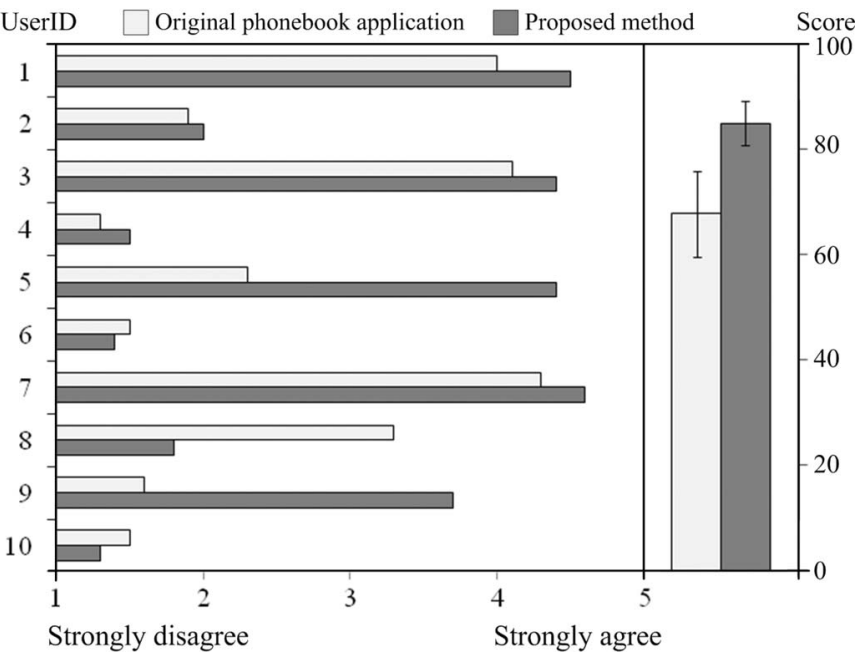

Fig. 12. SUS scores for the proposed system.

inal phonebook system. Although the SmartPhonebook gained a slightly less support in terms of simplicity (second and fourth statements), it was superior to the original one because of its functionality and convenience (fifth and eighth statements).

\section{CONCLUSiON}

Studying the communication patterns via the mobile phone gives strong insights into a subject's human relationships. This paper has proposed the SmartPhonebook which infers the user's personal and social contexts from uncertain mobile logs based on BNs and provides an effective way to contact someone and to manage social relationships by using a mobile phone. Moreover, it visualizes the social relations with icons and a network graph. The proposed system was tested on month-long mobile logs collected from ten users, and the feasibility was verified by implementing a workable prototype and by conducting the subjective test. A large-scale data set from the RM project was also used to evaluate the proposed system.

In order to model the accurate inference algorithm and to thoroughly evaluate it, it is required to collect a larger data set 
over a long period of time, with users' tagging their contexts, which is a challenging problem by itself. Here, the diversity modeling of the different life patterns such as sales people, consultants, and delivery personnel should be considered to improve the proposed method in terms of its generality and adaptability. One of the effective approaches in estimating a personalized model, which is capable of adapting to the diverse characteristics of the users, is to cluster the users into different groups and then to train an individual model inside each group, where the user's personality, environmental factors, and social circumstances could be employed as the features for the clustering. We will carry out these issues as our future work. Moreover, we will study on the context sharing among multiple users, where they interact with each other in estimating a realistic social network.

\section{REFERENCES}

[1] R. Ling, The Mobile Connection: The Cell Phone's Impact on Society. San Mateo, CA: Morgan Kaufmann, 2004.

[2] S. Menon and C. L. Horney, "Smartphone \& Chip Market Opportunities," Tempe, AZ, Rep. 9010, 2009.

[3] D. Bottazzi, R. Montanari, and A. Toninelli, "Context-aware middleware for anytime, anywhere social networks," IEEE Intell. Syst., vol. 22, no. 5, pp. 23-32, Sep./Oct. 2007.

[4] A. E. Milewski and T. M. Smith, "Providing presence cues to telephone users," in Proc. ACM Conf. Comput. Supported Cooperative Work, 2000, pp. 89-96.

[5] J. C. Tang, N. Yankelovich, J. Begole, M. V. Kleek, F. Li, and J. Bhalodia, "ConNexus to awarenex: Extending awareness to mobile users," in Proc. SIGCHI Conf. Human Factors Comput. Syst., 2001, pp. 221-228.

[6] A. Schmidt, A. Takaluoma, and J. Mntyjrvi, "Context-aware telephony over WAP," Pers. Technol., vol. 4, no. 4, pp. 225-229, Aug. 2000.

[7] A. Oulasvirta, R. Petit, M. Raento, and S. Tiitta, "Interpreting and acting on mobile awareness cues," Human-Comput. Interact., vol. 22, no. 1/2, pp. 97-135, May 2007.

[8] M. Raento, A. Oulasvirta, R. Petit, and H. Toivonen, "ContextPhone: A prototyping platform for context-aware mobile applications," IEEE Pervasive Comput., vol. 4, no. 2, pp. 51-59, Jan.-Mar. 2005.

[9] R.-H. Hwang, S.-Y. Tsai, and C.-Y. Wang, "UbiPhone: Human-centered ubiquitous phone system," IEEE Pervasive Comput., vol. 8, no. 2, pp. 4047, Apr--Jun. 2009.

[10] G. Mollenhorst, B. Volker, and H. Flap, "Social contexts and personal relationships: The effect of meeting opportunities on similarity for relationships of different strength," Social Netw., vol. 30, no. 1, pp. 60-68, Jan. 2008.

[11] N. Eagle, A. Pentland, and D. Lazer, "Inferring social network structure using mobile phone data," Proc. Nat. Acad. Sci., vol. 106, no. 36, pp. $15274-15278$, Sep. 2009.

[12] Y. Nakanishi, S. Kumazawa, T. Tsuji, and K. Hakozaki, "iCAMS2: Developing a mobile communication tool using location information and schedule information with J2ME," in Proc. MobileHCI, 2003, pp. 400-404.

[13] A. Burak and T. Sharon, "Analyzing usage of location based services," in Proc. CHI Extended Abstr. Human Factors Comput. Syst., 2003, pp. 970-971.

[14] F. Bentley and C. J. Metcalf, "The use of mobile social presence," IEEE Pervasive Comput., vol. 8, no. 4, pp. 35-41, Oct. 2009.

[15] E. Miluzzo, N. D. Lane, K. Fodor, R. Peterson, H. Lu, M. Musolesi, S. B. Eisenman, X. Zheng, and A. T. Campbell, "Sensing meets mobile social networks: The design, implementation and evaluation of the CenceMe application," in Proc. Int. Conf. Embedded Netw. Sens. Syst., 2008, pp. 337-350.

[16] K.-S. Hwang and S.-B. Cho, "Landmark detection from mobile life log using a modular Bayesian network model," Expert Syst. Appl., vol. 36, no. 10, pp. 12 065-12 076, Dec. 2009.

[17] J. Yang, "Toward physical activity diary: Motion recognition using simple acceleration features with mobile phones," in Proc. Int. Workshop Interactive Multimedia Consum. Electron., 2009, pp. 1-10.
[18] A. C. Santos, J. M. P. Cardoso, D. R. Ferreira, P. C. Diniz, and P. Chainho, "Providing user context for mobile and social networking applications," Pervasive Mobile Comput., vol. 6, no. 3, pp. 324-341, Jun. 2010.

[19] J.-H. Hong, S.-I. Yang, and S.-B. Cho, "ConaMSN: A context-aware messenger using dynamic Bayesian networks with wearable sensors," Expert Syst. Appl., vol. 37, no. 6, pp. 4680-4686, Jun. 2010.

[20] J. J. Jung, "Contextualized mobile recommendation service based on interactive social network discovered from mobile users," Expert Syst. Appl., vol. 36, no. 9, pp. 11950-11956, Nov. 2009.

[21] N. Eagle and A. Pentland, "Reality mining: Sensing complex social systems," Pers. Ubiquitous Comput., vol. 10, no. 4, pp. 255-268, Mar. 2006.

[22] Z. Shen and K. Ma, "MobiVis: A visualization system for exploring mobile data," in Proc. IEEE PacificVIS, 2008, pp. 175-182.

[23] A. Miklas, K. Gollu, K. Chan, S. Saroiu, K. Gummadi, and E. de Lara, "Exploiting social interactions in mobile systems," in Proc. 9th Int. Conf. Ubiquitous Comput., 2007, pp. 409-428.

[24] J.-K. Min, S.-H. Jang, and S.-B. Cho, "Mining and visualizing mobile social network based on Bayesian probabilistic model," in Proc. 6th Int. Conf. Ubiquitous Intell. Comput., 2009, pp. 111-120.

[25] R. Picard, "Affective computing," MIT Media Lab., Cambridge, MA, Media Laboratory Perceptual Computing TR 321, 1995.

[26] E. T. Hall, The Silent Language. Garden City, NY: Anchor, 1973.

[27] F. V. Jensen, Introduction to Bayesian Networks. New York: SpringerVerlag, 1996.

[28] J. Pearl, Probabilistic Reasoning in Intelligent Systems: Networks of Plausible Inference. San Mateo, CA: Morgan Kaufman, 1988.

[29] A. Bangor, P. T. Kortum, and J. T. Miller, "An empirical evaluation of the system usability scale," Int. J. Human-Comput. Interact., vol. 24, no. 6 , pp. 574-594, Aug. 2008.

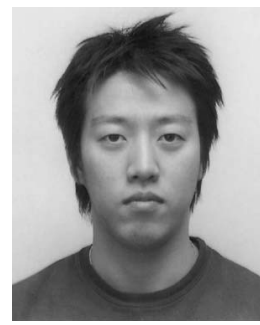

Jun-Ki Min received the B.S. and M.S. degrees in computer science from Yonsei University, Seoul, Korea, in 2004 and 2006, respectively, where he has been working toward the Ph.D. degree in the Department of Computer Science since 2006.

His research interests include pattern recognition, human-computer interaction, and mobile context awareness.

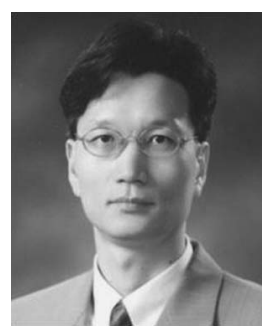

Sung-Bae Cho received the B.S. degree in computer science from Yonsei University, Seoul, Korea, in 1988, and the M.S. and Ph.D. degrees in computer science from Korea Advanced Institute of Science and Technology (KAIST), Daejeon, Korea, in 1990 and 1993, respectively.

From 1991 to 1993 , he was a member of the Research Team of the Center for Artificial Intelligence Research, KAIST. From 1993 to 1995, he was an Invited Researcher at the Human Information Processing Research Laboratories, Advanced Telecommunications Research Institute, Kyoto, Japan. In 1998, he was a Visiting Scholar at the University of New South Wales, Canberra, Australia. Since 1995, he has been an Associate Professor in the Department of Computer Science, Yonsei University. His research interests include neural networks, pattern recognition, intelligent man-machine interfaces, evolutionary computation, and artificial life.

Dr. Cho was a recipient of outstanding paper prizes from the IEEE Korea Section in 1989 and 1992 and another one from the Korea Information Science Society in 1990. He was also a recipient of the Richard E. Merwin Prize from the IEEE Computer Society in 1993, Best Paper Awards at the International Conference on Soft Computing in 1996 and 1998, and the Best Paper Award at the World Automation Congress in 1998. He was listed in Who's Who in Pattern Recognition from the International Association for Pattern Recognition in 1994, in Marquis Who's Who in Science and Engineering in 2000, and in Marquis Who's Who in the World in 2001. He is a member of the Korea Information Science Society, International Neural Network Society, IEEE Computer Society, and IEEE Systems, Man, and Cybernetics Society. 